\title{
Chaos Synchronization in Discrete-Time Dynamical Systems with Application in Population Dynamics
}

\author{
Tahmineh Azizi' ${ }^{1}$, Gabriel Kerr ${ }^{2}$ \\ ${ }^{1}$ Institute of Computational Comparative Medicine, Department of Anatomy and Physiology, Department of \\ Mathematics, Kansas State University, Manhattan, KS, USA \\ ${ }^{2}$ Department of Mathematics, Kansas State University, Manhattan, KS, USA \\ Email: tazizi@ksu.edu
}

How to cite this paper: Azizi, T. and Kerr, G. (2020) Chaos Synchronization in Discrete-Time Dynamical Systems with Application in Population Dynamics. Journal of Applied Mathematics and Physics, 8, 406-423.

https://doi.org/10.4236/jamp.2020.83031

Received: January 22, 2020

Accepted: February 25, 2020

Published: February 28, 2020

Copyright $\odot 2020$ by author(s) and Scientific Research Publishing Inc. This work is licensed under the Creative Commons Attribution International License (CC BY 4.0).

http://creativecommons.org/licenses/by/4.0/

\begin{abstract}
Study of chaotic synchronization as a fundamental phenomenon in the nonlinear dynamical systems theory has been recently raised many interests in science, engineering, and technology. In this paper, we develop a new mathematical framework in study of chaotic synchronization of discrete-time dynamical systems. In the novel drive-response discrete-time dynamical system which has been coupled using convex link function, we introduce a synchronization threshold which passes that makes the drive-response system lose complete coupling and synchronized behaviors. We provide the application of this type of coupling in synchronized cycles of well-known Ricker model. This model displays a rich cascade of complex dynamics from stable fixed point and cascade of period-doubling bifurcation to chaos. We also numerically verify the effectiveness of the proposed scheme and demonstrate how this type of coupling makes this chaotic system and its corresponding coupled system starting from different initial conditions, quickly get synchronized.
\end{abstract}

\section{Keywords}

Chaos Synchronization, Synchronization Threshold, Period-Doubling Bifurcation, Convex Link Function, Nonlinear Dynamics

\section{Lead Paragraph}

Synchronization of complex population oscillations in natural systems has been examined widely by many researchers. Due to the sensitivity on initial conditions of chaotic systems, synchronization of chaotic systems does not look to be 
possible. However, it has been shown that it is still possible that under some conditions, two synchronized systems in chaotic regime get synchronized again. There are many studies about how we can couple continuous time dynamical systems but we cannot find any explicit and concrete way to couple the discrete-time dynamical systems. Since discrete-time systems play important role in modeling non-overlapping generations, we have developed a novel way to couple these systems which keeps the same qualitative dynamics and we have provided one well known example of this type of coupling. Also, we have defined a new concept in the area of chaos synchronization called synchronization threshold. By passing the threshold both drive and response system lose the condition for complete synchronization and their solutions diverge from each other.

\section{Introduction}

Population dynamics can be modeled through the continuous-time system and the discrete-time system. However, when population size is small or that population does not overlap, discrete-time system is more appropriate to use. Discrete-time population models are widely used to describe the dynamics of hosts and parasitoids interactions [1] [2] [3]. There are many simple nonlinear discrete-time biological models which create rich and complex spectrum of dynamics from coexistence of species through periodic cycles to irregular and chaotic behaviors [4] [5] [6] [7] [8]. Chaos and synchronization are two widespread phenomena with application in many disciplines which have been considered as a central topic in nonlinear dynamics [9] [10] [11]. The presence of chaos in population models has been extensively reported by different researchers [4] [5] [6] [7] [8]. The main property of chaotic dynamics is its critical sensitivity to initial conditions, which is responsible for initially neighboring trajectories separating from each other exponentially in the course of time. Synchronization implies that there is a strong correlation between coupled systems and intuitively, it refers to a phenomenon which makes the systems have the same dynamical behavior. Traditionally, synchronization was based upon periodic signals. However, after coming to the chaotic signals, more possibilities and flexibilities have been entered in this area.

Chaos synchronization, has been started by the work of Fujisaka and Yamada [12] in 1983. After 1990, when the possibility of chaos synchronization was understood by researchers, this idea has received many attractions by people in different areas [13] [14] [15] [16] [17]. Synchronized chaos means that for any two chaotic systems for which any two nearby initial points in phase space quickly diverge and become unpredictable, it is possible that these two converge toward each other and evolve with each other in time. Complete synchronization takes place if there is a perfect linking of the chaotic solutions such that they remain in step with each other in time. In 1990, L.M. Pecora and L. Carroll, described a coupling method which constructs a real set of chaotic synchronization circuits [13]. They have applied this common signal to several well-known continuous-time dynamical systems such as Lorenz and Rossler and they claimed 
that it is possible to use this method with a slight variation for discrete-time dynamical systems. Chaos synchronization has great interest and application in different disciplines like physics [9] and biology [10], and it has been observed in a huge variety of phenomena in nature [11]. Synchronization has an important role in self-organization of organisms' groups in various biological systems [18]. There are several types of synchronization, such as complete synchronization [14], generalized synchronization [19] [20] [21], phase synchronization [22], lag synchronization [23], antisynchronization and projective synchronization [24] and [25].

This study proposes a new way to couple of discrete-time dynamical systems. We study three different types of synchronization for this new coupled system and we present the results related to the local stability of drive response system and we find the attractive set of this novel coupled system. This paper also studies the interesting dynamics of a drive-response Ricker model which has been coupled by convex link function. Our goal is to build an appropriate response system which traces the drive system and finally they evolve in time even in chaotic regime. We explain that how this coupling method can be applied on a general discrete-time dynamical system to get a complete synchronization. Finally, the long term analysis through bifurcation diagrams and also time-series analysis exhibit that this drive-response system which reveals complex dynamics including cascade of period doubling to chaotic solutions, for smaller synchronization threshold, get completely synchronized.

\section{Description of the Coupling Method}

In this section, we study the complete synchronization in a general discrete-time drive-response system. Here, we use a convex function to build the proposed drive-response system. To begin with, consider the following discrete-time dynamical system:

$$
X_{n+1}=f\left(X_{n}\right)
$$

where $X \in \mathbb{R}^{n}$ is the state vector of drive system at time $n, f$ is a mapping from $\mathbb{R}^{n}$ to itself and is continuously differentiable. Next step is to find a perfect linking such that the system (2.1) and new coupled one remain in step with each other in time. To model the response system or coupled system, we use a convex link function as the form $H(X, Y):=(1-s) X+s Y$ where $H: \mathbb{R}^{2 n} \rightarrow \mathbb{R}^{n}$ and $X, Y \in \mathbb{R}^{n}$ are the state vectors of response system at time $n$, and $0<s \leq 1$ is synchronization threshold. Therefore, for $H_{n}:=(1-s) X_{n}+s Y_{n}$, the response system has the form:

$$
Y_{n+1}:=f\left(H_{n}\right)=f\left((1-s) X_{n}+s Y_{n}\right)
$$

and we demonstrate the error between the solutions of the drive system (2.1) and the response system (2.2) by $e(n)=\left\|Y_{n}-X_{n}\right\|$.

\subsection{Complete Synchronization Using Contraction Mapping Theorem}

To explain the complete synchronization between two systems (2.1) and (2.2), 
we need to recall some known concepts which are crucial part of the proposed coupling method:

Definition 2.1 We say that the drive system (2.1) and response system (2.2) are in complete synchronization if

$$
\lim _{n \rightarrow \infty} e(n)=\lim _{n \rightarrow \infty}\left\|Y_{n}-X_{n}\right\|=0
$$

means that two systems eventually evolve identically in time.

Definition 2.2 Let $E$ be a Banach space. Then, the map $F: E \rightarrow E$ is called a contraction mapping if there exists a constant $0 \leq \alpha<1$ such that for every pair of points $X, Y \in E$, we have $\|F(X)-F(Y)\| \leq \alpha\|X-Y\|$, where $\alpha$ is called a contraction constant of $F$ on $E$ [26].

The error between the drive and response system (2.1) and (2.2) has the following form:

$$
e(n+1)=Y_{n+1}-X_{n+1}=f\left((1-s) X_{n}+s Y_{n}\right)-f\left(X_{n}\right)
$$

We can easily see that for $0<s \leq 1$ :

$$
\left\|\left((1-s) X_{n}+s Y_{n}\right)-X_{n}\right\| \leq s\left\|Y_{n}-X_{n}\right\|
$$

Here, we assume that $f$ is a contraction mapping. Then, for the Equation (2.4) we can write:

$$
\|e(n+1)\|=\left\|f\left((1-s) X_{n}+s Y_{n}\right)-f\left(X_{n}\right)\right\| \leq \beta\left\|Y_{n}-X_{n}\right\|=\beta\|e(n)\|
$$

where, $\beta$ is a contraction constant.

As we defined before, to get complete synchronization, we need to have $\lim _{n \rightarrow \infty}\|e(n)\|=0$. Therefore, for contraction constant $0 \leq \beta<1$,

$$
\lim _{n \rightarrow \infty}\|e(n+1)\|=\lim _{n \rightarrow \infty}\left\|Y_{n+1}-X_{n+1}\right\|=0
$$

which means that the drive-response system (2.1)-(2.2) satisfies the complete synchronization properties. We will find $\beta$ in theorem (2.3).

Theorem 2.3 Given the non-linear coupled dynamical system (2.1) and (2.2), where the map $f: \mathbb{R}^{2 n} \rightarrow \mathbb{R}^{n}$, and for the values $s<\tilde{s}=\frac{1}{\rho_{A}+\alpha}$, we get

$$
\lim _{n \rightarrow \infty}\left\|Y_{n+1}-X_{n+1}\right\|=0
$$

means that passing the synchronization threshold $\tilde{s}$ makes the drive-response system (2.1) and (2.2) lose the complete synchronization properties.

Proof. Suppose the following $C^{r}$ maps which have a fixed point at the origin:

$$
\begin{gathered}
X_{n+1}=A X_{n}+F\left(X_{n}\right), \\
Y_{n+1}=A\left((1-s) X_{n}+s Y_{n}\right)+F\left((1-s) X_{n}+s Y_{n}\right),
\end{gathered}
$$

where the contraction mapping $F\left(X_{n}\right)=F_{2}\left(X_{n}\right)+\cdots+F_{r-1}\left(X_{n}\right)+O\left(\left|\left(X_{n}\right)\right|^{r}\right)$, includes the vector-valued homogeneous polynomials of degree $2, \cdots, r$. Con- 
sider the following equation for the error:

$$
\begin{aligned}
e(n+1) & =Y_{n+1}-X_{n+1} \\
& =(1-s) A X_{n}+s A Y_{n}-A X_{n}+F\left((1-s) X_{n}+s Y_{n}\right)-F\left(X_{n}\right) \\
& =s A\left(Y_{n}-X_{n}\right)+F\left((1-s) X_{n}+s Y_{n}\right)-F\left(X_{n}\right)
\end{aligned}
$$

Since, we assumed that $F$ is a contraction mapping, it satisfies the following inequality:

$$
\|F(Y)-F(X)\| \leq \alpha\|Y-X\|
$$

where, $\alpha$ is a contraction constant. By triangular inequality we can write:

$$
\begin{aligned}
\|e(n+1)\| & =\left\|s A\left(Y_{n}-X_{n}\right)+F\left((1-s) X_{n}+s Y_{n}\right)-F\left(X_{n}\right)\right\| \\
& \leq s \rho_{A}\left\|Y_{n}-X_{n}\right\|+s \alpha\left\|Y_{n}-X_{n}\right\| \\
& =s \rho_{A}\|e(n)\|+s \alpha\|e(n)\|
\end{aligned}
$$

where, $0<s \leq 1$ and $\rho_{A}$ is the spectral radius of $A$ which is equal to $\rho_{A}=\max \left|\lambda_{i}\right|$ where $\lambda$ is the root of characteristic polynomial or eigenvalue for $A$. Since, $0 \leq \alpha<1$, therefore

$$
\|e(n+1)\| \leq s \rho_{A}\|e(n)\|+s \alpha\|e(n)\|=s\left(\rho_{A}+\alpha\right)\|e(n)\|
$$

We know that for complete synchronization, the error between the solutions should converge toward zero. Thus, $\lim _{n \rightarrow \infty}\|e(n)\|=\lim _{n \rightarrow \infty}\left\|Y_{n}-X_{n}\right\|=0$. As a result, for $s\left(\rho_{A}+\alpha\right)<1$ we have

$$
\lim _{n \rightarrow \infty}\|e(n+1)\|=\lim _{n \rightarrow \infty}\left\|Y_{n+1}-X_{n+1}\right\|=0
$$

for which, $s<\frac{1}{\rho_{A}+\alpha}=\tilde{s}$. Here, $\tilde{s}=\beta$, which we discussed in the beginning of this section. After passing $\tilde{s}$, we lose the complete synchronization between (2.1) and (2.2).

Remark 1 Consider the drive system (2.1) becomes periodic with period $\ell$, i.e., $X_{n+\ell}=X_{n}$. Then, for the values $s<\frac{1}{\rho_{A}+\alpha}$, the non-linear coupled $d y$ namical system (2.1) and (2.2) become completely synchronized. In other word,

$$
\lim _{n \rightarrow \infty}\left\|Y_{n+1}-X_{n+1}\right\|=0
$$

Here, similar to the proof of theorem (2.3), we decompose the nonlinear dynamical system (2.1) and (2.2) into linear and non-linear part. In this case, using triangular inequality we have:

$$
\|e(n+1)\| \leq\left(s\left(\rho_{A}+\alpha\right)\right)^{\ell}\|e(n)\|
$$

We know that

$$
\lim _{n \rightarrow \infty}\|e(n)\|=\lim _{n \rightarrow \infty}\left\|Y_{n}-X_{n}\right\|=0
$$

Therefore, for $\left(s\left(\rho_{A}+\alpha\right)\right)^{\ell}<1$ we have

$$
\lim _{n \rightarrow \infty}\|e(n+1)\|=0
$$


for which, $s<\frac{1}{\rho_{A}+\alpha}=\tilde{s}$.

\subsection{Local Dynamics, Attractors and Attracting Set of Drive-Response System}

We continue this section by seeking appropriate closed subset $\Omega \in \mathbb{R}^{n}$ in which for drive and response system (2.1) and (2.2); where $X=\left(X_{0}, X_{1}, \cdots, X_{n}\right)$ and $Y=\left(Y_{0}, Y_{1}, \cdots, Y_{n}\right)$, the following conditions hold:

1) for all $X, Y \in \Omega$, then $f(X) \in \Omega, f(H) \in \Omega \times \Omega$.

2) $f$ is a contraction on $\Omega$.

The Jacobian matrix for drive-response system (2.1)-(2.2) has the following form:

$$
J:=\left[\begin{array}{cc}
\frac{\partial f(X)}{\partial X} & 0 \\
\frac{\partial f(H)}{\partial X} & \frac{\partial f(H)}{\partial Y}
\end{array}\right]
$$

We can immediately obtain the following result:

Proposition 2.4 Given Jacobian matrix (2.7), for which the following inequality holds:

$$
\|J\|:=\rho_{J}=\max \left|\lambda_{i}\right| \leq \beta<1
$$

where $\beta$ is the contraction constant, $\rho_{J}$ is the spectral radius of $J$ and $\lambda_{i}$ for $i=1, \cdots, n$ are the eigenvalues of Jacobian matrix J. Then, the mapping $f$ is a contraction and the drive-response system (2.1)-(2.2) satisfies the complete synchronization properties, i.e.

$$
\lim _{n \rightarrow \infty}\|e(n+1)\|=\lim _{n \rightarrow \infty}\left\|Y_{n+1}-X_{n+1}\right\|=0
$$

Proof. By contraction mapping theorem. Assume that the eigenvalues of the Jacobian matrix (2.7) have absolute values less than one. Then, using the contraction mapping theorem, $f$ satisfies the contraction properties and would be a contraction mapping. Therefore, for the Equation (2.4) we can write:

$$
\|e(n+1)\|=\left\|f\left((1-s) X_{n}+s Y_{n}\right)-f\left(X_{n}\right)\right\| \leq \beta\left\|Y_{n}-X_{n}\right\|=\beta\|e(n)\|
$$

where, $\beta$ is a contraction constant. Thus, for contraction constant $0 \leq \beta<1$,

$$
\lim _{n \rightarrow \infty}\|e(n+1)\|=\lim _{n \rightarrow \infty}\left\|Y_{n+1}-X_{n+1}\right\|=0
$$

which means that the drive-response system (2.1)-(2.2) satisfies the complete synchronization properties.

The schematic representation for this type of coupling to obtain complete synchronization has been demonstrated in Figure 1.

Recall from the theorem (2.3) that the linearization of a given autonomous drive-response problem discrete dynamical system can be written as the form:

$$
Z_{n+1}:=h\left(Z_{n}\right)=J Z_{n}+\tilde{h}\left(Z_{n}\right),
$$




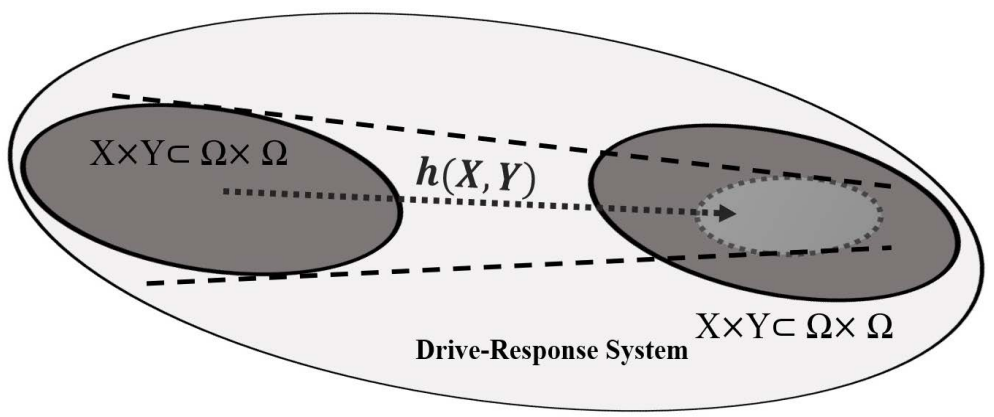

Figure 1. The schematic diagram for complete synchronization in a discrete-time drive-response dynamical system.

where, $Z=(X, Y)$ and $h: \mathbb{R}^{2 n} \rightarrow \mathbb{R}^{2 n}$ is a sufficiently smooth governing transition function.

Remark 2. For drive-response system (2.9), the following hold:

1) For all $Z \in \Omega \times \Omega$, then $h(Z) \in \Omega \times \Omega$.

2) $h$ is a contraction on $\Omega \times \Omega$.

Therefore, we have the following statements for system (2.9).

Proposition 2.5 Consider the drive-response system $h: \mathbb{R}^{2 n} \rightarrow \mathbb{R}^{2 n}$ which is defined on a closed subset $\Omega \times \Omega \subset \mathbb{R}^{2 n}$ and satisfies the conditions in remark (2.2). Then, there exists a unique $Z^{*}$ with $h\left(Z^{*}\right)=Z^{*}$. In another word, if (2.8) holds, then the drive-response system (2.9) has a unique fixed point.

Remark 3 According to the well known contraction mapping theorem, the converse of proposition (2.5) does not necessarily hold.

For drive-response system (2.9), we can establish notions of sequential convergence and hence of stability for drive-response system (2.9). We now provide a general theorem which is the result of proposition (2.4).

Theorem 2.6 Let $h: \mathbb{R}^{2 n} \rightarrow \mathbb{R}^{2 n}$ be a continuously differentiable map defined on a closed subset $\Omega \times \Omega \subset \mathbb{R}^{2 n}$ and let J satisfying the form (2.7) be the Jacobian matrix of drive-response system (2.9) with $\left|\frac{\partial f(X)}{\partial X}\right|<1$ and $\left|\frac{\partial f(H)}{\partial Y}\right|<1$. Then, the following hold:

1) The solutions $Z=(X, Y) \in \Omega \times \Omega$ of drive-response system (9) completely synchronized.

2) Equilibrium $Z^{*}$ of drive-response system (9) is stable, i.e.

for any $\varepsilon>0$, there exists $\delta>0$ such that $\left\|Z^{*}-Z\right\|<\delta$ implies that $\left\|h^{\ell}(Z)-Z^{*}\right\|<\varepsilon$ for $\ell>0$.

Proof. The proof is straightforward.

Remark 4 In theorem (2.6), hyperbolicity is a robust property and it is one of the most important assumptions.

It has been experimentally observed that there may be some situations in which the response system is stable but the response system has complex dynamics and the reason is using non differentiable link function or any 
non-differentiable transport system. In this study, we take the advantages of using a continuous convex link function which can completely control over the behavior of response system and we will numerically show that the response system inherits the same qualitative dynamics as its drive system and even for smaller synchronization threshold, the response system and drive system are almost completely equivalent.

\section{Application of Chaos Synchronization in Population Dynamics}

The chaotic behavior may be observed experimentally in natural systems in many scientific areas. Chaos can be defined as irregular and unpredictable time evolution of non linear systems. Main characteristic of chaos is sensitivity on initial conditions and that system does not repeat its past behavior. Despite the fact that chaotic systems are sensitive to initial conditions, it has been experimentally shown that the chaotic oscillators could be coupled. Chaos synchronization occurs when a chaotic oscillator drives another chaotic oscillator and is a very important phenomenon which has been occurred widely in ecological systems [27] [28] [29] [30]. Because of importance of synchronization and its consequences on population dynamics, we study Ricker model and its synchronized system and we present their qualitative dynamics using different dynamical system tools.

The Ricker model is one of the most widely-used ecological models which displays regular and irregular complex nonlinear dynamics [31] [32] and its coupled system as the following form:

$$
\begin{gathered}
R_{1}:=x_{1}(n+1)=x_{1}(n) \mathrm{e}^{r\left(1-\frac{x_{1}(n)}{k}\right)} \\
R_{2}:=x_{2}(n+1)=H \mathrm{e}^{r\left(1-\frac{H}{k}\right)}
\end{gathered}
$$

where

$$
H\left(x_{1}, x_{2}\right):=(1-s) x_{1}+s x_{2}
$$

Here, $x_{1}$ demonstrates the population size of drive system, $x_{2}$ represents the population size of response system, $r$ is the intrinsic growth rate, $k$ is the carrying capacity of the environment, $s \in(0,1]$ is synchronization threshold and $H\left(x_{1}, x_{2}\right): \mathbb{R}^{2} \rightarrow \mathbb{R}$ is a link function which has been used to couple (3.2) and (3.2). Thus, if drive system $R_{1}: \mathbb{R} \rightarrow \mathbb{R}$, the corresponding response system would be $R_{2}: \mathbb{R}^{2} \rightarrow \mathbb{R}$, where $H$ is a function of $x_{1}$ and $x_{2}$.

For drive-response (3.2)-(3.2), the steady states are $(0,0),\left(0, x_{2}^{*}\right)$, and $(k, k)$. The Jacobian matrix for (3.1)-(3.2) has the form

$$
J:=\left[\begin{array}{cc}
\frac{\partial R_{1}}{\partial x_{1}} & 0 \\
\frac{\partial R_{1}}{\partial x_{1}} & \frac{\partial R_{1}}{\partial x_{2}}
\end{array}\right]
$$


where

$$
\begin{gathered}
\frac{\partial R_{1}}{\partial x_{1}}=\left(1-\frac{r x_{1}}{k}\right) \exp \left(r\left(1-\frac{x_{1}}{k}\right)\right) \\
\frac{\partial R_{1}}{\partial x_{1}}=(1-s) \exp \left(r\left(1-\frac{(1-s) x_{1}+s x_{2}}{k}\right)\right)\left(1-\frac{\left((1-s) x_{1}+s x_{2}\right) r}{k}\right) \\
\frac{\partial R_{1}}{\partial x_{2}}=s \exp \left(r\left(1-\frac{(1-s) x_{1}-s x_{2}}{k}\right)\right)\left(1-\frac{(1-s) x_{1}+s x_{2}}{k}\right)
\end{gathered}
$$

Then, at the origin we have

$$
\left.J\right|_{(0,0)}=\left(\begin{array}{cc}
\mathrm{e}^{r} & 0 \\
(1-s) \mathrm{e}^{r} & s \mathrm{e}^{r}
\end{array}\right)
$$

and for the positive fixed point $(k, k)$ we have

$$
\left.J\right|_{(k, k)}=\left(\begin{array}{cc}
1-r & 0 \\
1-s-r+r s & s-r s
\end{array}\right)
$$

Also, for $\left(0, x_{2}^{*}\right)$,

$$
\left.J\right|_{\left(0, x_{2}^{*}\right)}=\left(\begin{array}{c}
\mathrm{e}^{r} \\
\left.(1-s) \exp \left(r\left(1-\frac{x_{2}^{*}}{k}\right)\right)\left(1-\frac{x_{2}^{*} r}{k}\right) \operatorname{sexp}\left(r\left(1-\frac{x_{2}^{*}}{k}\right)\right)\left(1-\frac{x_{2}^{*}}{k}\right)\right)
\end{array}\right.
$$

Proposition 3.1 The local stability analysis results for the fixed points of (3.1)-(3.2) are summarized as below.

1) The equilibrium point $(0,0)$ for $r<-\ln s$ is a saddle point, and for $r>-\ln s$ is an unstable fixed point.

2) The equilibrium point $(k, k)$ is a stable point in the interior of positive quadratic if $s<1 /(1-r), 0<r<1$ and $s>1 /(1-r), 1<r<2$, or $s<1 /(r-1), 0<r<1$ and $s<-1 /(1-r), 1<r<2 .(k, k)$ is a saddle point if $s>1 /(1-r), r>2$ or $s<-1 /(1-r), r>2$.

3) The equilibrium point $\left(0, x_{2}^{*}\right)$ for $s>k \exp \left[r\left(x_{2}^{*}-k\right) / k\right] /\left(k-x_{2}^{*}\right)$ or $s<k \exp \left[r\left(x_{2}^{*}-k\right) / k\right] /\left(x_{2}^{*}-k\right)$ is an unstable point, and for $|s|<k \exp \left[r\left(x_{2}^{*}-k\right) / k\right] /\left(k-x_{2}^{*}\right)$ is a saddle point.

To study the global stability of the equilibrium points of both systems, at first we prove that all solutions in the first quadrant $\mathbb{R}_{+}^{2}$ are eventually bounded.

Theorem 3.2 For $r>0, k>0$ and initial conditions in the first quadrant $\mathbb{R}_{+}^{2}$, i.e. $x_{1}(0)>0$ and $x_{2}(0)>0$, for system of (3.1)-(3.2) we have: $x_{1}>0$ and $x_{2}>0$ for all $n \in \mathbb{Z}^{+}$. In addition, we can find some positive number $M$, such that $\max _{n \in \mathbb{Z}^{+}}\left\{x_{1}(n), x_{2}(n)\right\} \leq M$.

Proof. By induction.

Since $x_{1}(0)>0$ we have $\exp \left(r\left(1-\frac{x_{1}(0)}{k}\right)\right)>0$, hence

$$
x_{1}(1)=x_{1}(0) \mathrm{e}^{r\left(1-\frac{x_{1}(0)}{k}\right)}>0
$$


Assume that for $n \leq l$, we have $x_{1}(l)>0$. Then for $n=l+1$ we have

$$
x_{1}(l+1)=x_{1}(l) \mathrm{e}^{r\left(1-\frac{x_{1}(l)}{k}\right)}>0
$$

Therefore $x_{1}(n)>0$ for any $n \in \mathbb{Z}^{+}$. Similarly, since $x_{1}(0)>0$ and $x_{2}(0)>0$, we have $\mathrm{e}^{r\left(1-\frac{(1-s) x_{1}(0)+s x_{2}(0)}{k}\right)}$ is positive. Hence,

$$
x_{2}(1)=\left((1-s) x_{1}(0)+s x_{2}(0)\right) \mathrm{e}^{r\left(1-\frac{(1-s) x_{1}(0)+s x_{2}(0)}{k}\right)}>0
$$

Assume that for $n \leq l$, we have $x_{2}(l)>0$. Then for $n=l+1$ we have

$$
x_{2}(l+1)=\left((1-s) x_{1}(l)+s x_{2}(l)\right) \mathrm{e}^{r\left(1-\frac{(1-s) x_{1}(l)+s x_{2}(l)}{k}\right)}>0
$$

Therefore $x_{2}(n)>0$ for any $n \in \mathbb{Z}^{+}$. To find an upper bound, we know,

$$
x_{1}(n+1)=x_{1}(n) \mathrm{e}^{r\left(1-\frac{x_{1}(n)}{k}\right)} \leq \max _{x \in \mathbb{R}^{+}}\{f(x)\}
$$

If we define $f(x)=x \mathrm{e}^{r\left(1-\frac{x}{k}\right)}$, then $f^{\prime}(x)=\left(1-\frac{r x}{k}\right) \mathrm{e}^{r\left(1-\frac{x}{k}\right)}$ and $f(x)$ has critical points at $x=\frac{k}{r}$. Since $f^{\prime}(x)>0$ if $x<\frac{k}{r}$ and $f^{\prime}(x)<0$ if $x>\frac{k}{r}$, then $x=\frac{k}{r}$ is the maximal point of $f(x)$, i.e. $\max _{x \in \mathbb{R}^{+}}\{f(x)\}=f\left(\frac{k}{r}\right)$. Hence,

$$
x_{1}(n+1)=x_{1}(n) \mathrm{e}^{r\left(1-\frac{x_{1}(n)}{k}\right)} \leq \max _{x \in \mathbb{R}^{+}}\left\{x \mathrm{e}^{r\left(1-\frac{x}{k}\right)}\right\}=f\left(\frac{k}{r}\right)=\frac{k \mathrm{e}^{r-1}}{r}=M
$$

Similarly,

$$
\begin{aligned}
x_{2}(n+1) & =\left((1-s) x_{1}(n)+s x_{2}(n)\right) \mathrm{e}^{r\left(1-\frac{(1-s) x_{1}(n)+s x_{2}(n)}{k}\right)} \\
& =H \mathrm{e}^{r\left(1-\frac{H}{k}\right)} \leq \max _{x \in \mathbb{R}^{+}}\left\{H \mathrm{e}^{r\left(1-\frac{H}{k}\right)}\right\}=\frac{k \mathrm{e}^{r-1}}{r}=M
\end{aligned}
$$

Therefore, we can find some positive number $M$, such that $\max _{n \in \mathbb{Z}^{+}}\left\{x_{1}(n), x_{2}(n)\right\} \leq M$.

Theorem 3.3 If there are positive constants $m>0$ and $M>0$ such that the solution $\left(x_{1}(n), x_{2}(n)\right)$ of system satisfies

$$
\begin{aligned}
& 0<m \leq \lim _{n \rightarrow+\infty} \inf x_{1}(n) \leq \lim _{n \rightarrow+\infty} \sup x_{1}(n) \leq M=\frac{k \mathrm{e}^{r-1}}{r} \\
& 0<m \leq \lim _{n \rightarrow+\infty} \inf x_{2}(n) \leq \lim _{n \rightarrow+\infty} \sup x_{2}(n) \leq M=\frac{k \mathrm{e}^{r-1}}{r}
\end{aligned}
$$

Then, system (3.1)-(3.2) is persistent. If system is not persistent, it is called non-persistent [33]. 
Theorem 3.4 Given $r>0, k>0$ and initial conditions $x_{1}(0)>0$ and $x_{2}(0)>0$ in system (3.1)-(3.2), if the following conditions hold:

1) $\left|\left(1-\frac{r x_{1}}{k}\right) \exp \left(r\left(1-\frac{x_{1}}{k}\right)\right)\right|<1$

2) $\left|s\left(1-\frac{(1-s) x_{1}+s x_{2}}{k}\right) \exp \left(r\left(1-\frac{(1-s) x_{1}-s x_{2}}{k}\right)\right)\right|<1$

Then for drive-response system (3.1)-(3.2) for $\left(x_{1}, x_{2}\right) \in \Omega \times \Omega$ we have,

$$
\lim _{n \rightarrow \infty}\|e(n+1)\|=\lim _{n \rightarrow \infty}\left\|x_{2}(n+1)-x_{1}(n+1)\right\|=0
$$

where

$$
\Omega \times \Omega=\left\{\left(x_{1}, x_{2}\right) \mid \max _{x \in \mathbb{Z}^{+}}\left\{x_{1}(n), x_{2}(n)\right\} \leq \frac{k \mathrm{e}^{r-1}}{r}\right\}
$$

Proof. Using theorem (2.6), since $\Omega \times \Omega$ is closed and the conditions of proposition (2.4) are satisfied, therefore the solutions of drive-response system (3.1)-(3.2) are completely synchronized and the error between the solutions converges toward zero.

\subsection{Phase and Amplitude Synchronization in Population Dynamics}

We begin with two important concepts in theory of synchronization of chaotic systems: a) mean phase difference, b) mean amplitude difference. We analyze these two types of synchronizations for drive-response population model (3.1)-(3.2) which have been coupled using the proposed link function. We consider the oscillations of this discrete-time population system as being synchronized if their phases coincide repeatedly and they have identical mean amplitude. These two types of synchronization have been studied widely in science, nature, engineering, or social life [34]-[39] and before using them, we briefly define them.

Definition 3.5 We call two systems are in phase synchronization if they have equivalent mean phase or they have a constant difference in phase. We define mean phase for two oscillators as

$$
\Phi_{n} \equiv\left\|X_{n+1}-X_{n}\right\| \Rightarrow\left\langle\Phi_{\tau}\right\rangle=\frac{\sum_{n=1}^{N_{\tau}} \Phi_{n}}{N_{\tau}}
$$

where, $N_{\tau}$ is the number of cycles within a time $\tau$.

In fact, for two non-identical oscillators, phase synchronization happens when their phases evolve in synchrony but their amplitude remain unsynchronized.

Definition 3.6 We call two systems are in amplitude synchronization if they have identical mean amplitude. We define mean amplitude for two oscillators as

$$
\langle A\rangle=\frac{\sum_{n=1}^{N_{\tau}} A_{n}}{N_{\tau}}
$$

Remark 5 The mean amplitude and mean phase are qualitatively similar with 


\section{each other.}

Figure 2 demonstrates the mean phase difference, i.e. $\left|\Phi_{R_{2}}-\Phi_{R_{1}}\right|$ and the mean amplitude difference, i.e. $\left|A_{R_{2}}-A_{R_{1}}\right|$ corresponding to Ricker model and its synchronized model (3.1) and (3.2). As we can see, using these two tools, we can numerically catch the threshold at which the systems (3.1) and (3.2) satisfy the phase synchronization and amplitude synchronization properties.

\subsection{Complete Synchronization in Population Dynamics}

In this section, we study the complete synchronization for drive-response system (3.1)-(3.2) using some qualitative methods which have been used frequently to detect chaos. In order to understand some dynamical behaviors of systems including period doubling bifurcations and chaotic oscillations, we picked a single parameter bifurcation, which can demonstrate how dependence is the dynamics of the systems on a certain parameter.

Figure 3 demonstrates the solutions of drive system (3.1) (red color) and response system (3.2) (black color) with different initial conditions and some interesting $r$ values while $s=0.5$.

For $r=1.3$ and $s=0.5$ both drive and response system (3.1)-(3.2) evidently, exhibit the sigmoidal approach to carrying capacity reminiscent of the logistic model. For $r=1.9$, we have damped oscillations toward steady state which is because of two biological phenomena at first, the population which started below carrying capacity does not smoothly approach steady state through a phenomenon called as overcompensation and then this follows by the second phenomenon called undershooting, which is due to further overshooting. If we increase $r$ further, for the value $r=2.1$ we see that these damped oscillations follows by a 2 -cycle pattern and they are diverging from the steady states. Similarly, for $r=2.2$ and $r=2.4$, the oscillations follow a 2-cycle pattern. Finally, for $r=3.8$ we can see the occurrence of unpredictable, irregular and chaotic oscillations.

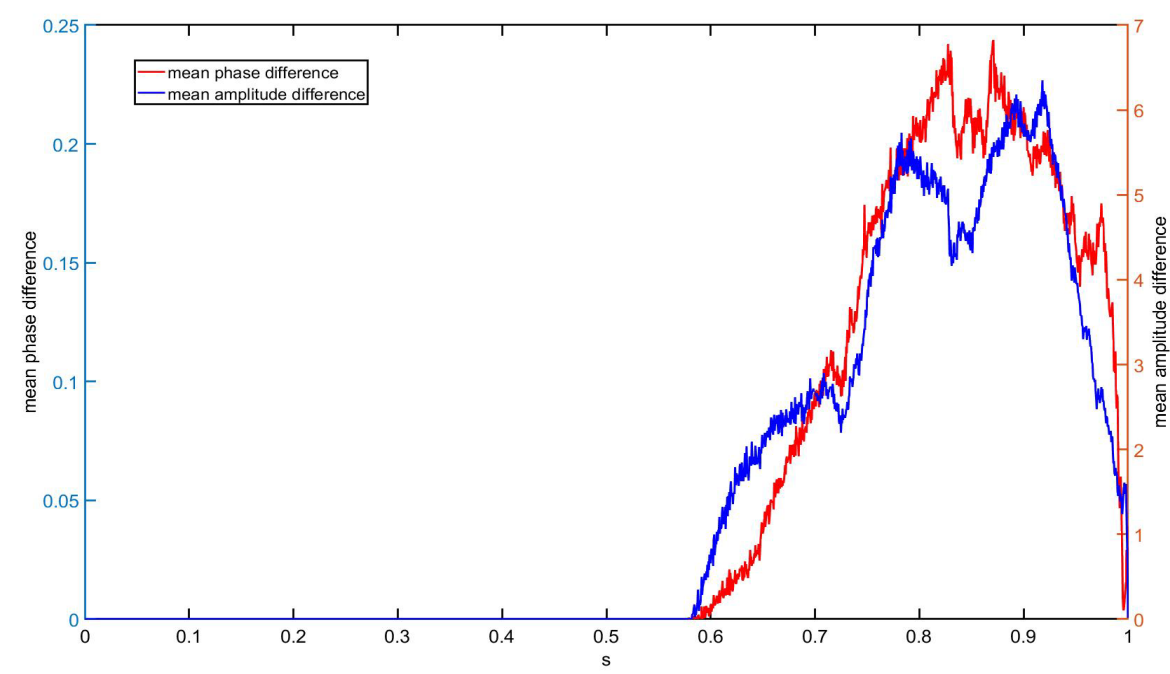

Figure 2. The mean phase difference and the mean amplitude difference for drive-response system (3.1)-(3.2) when $r=3$. 

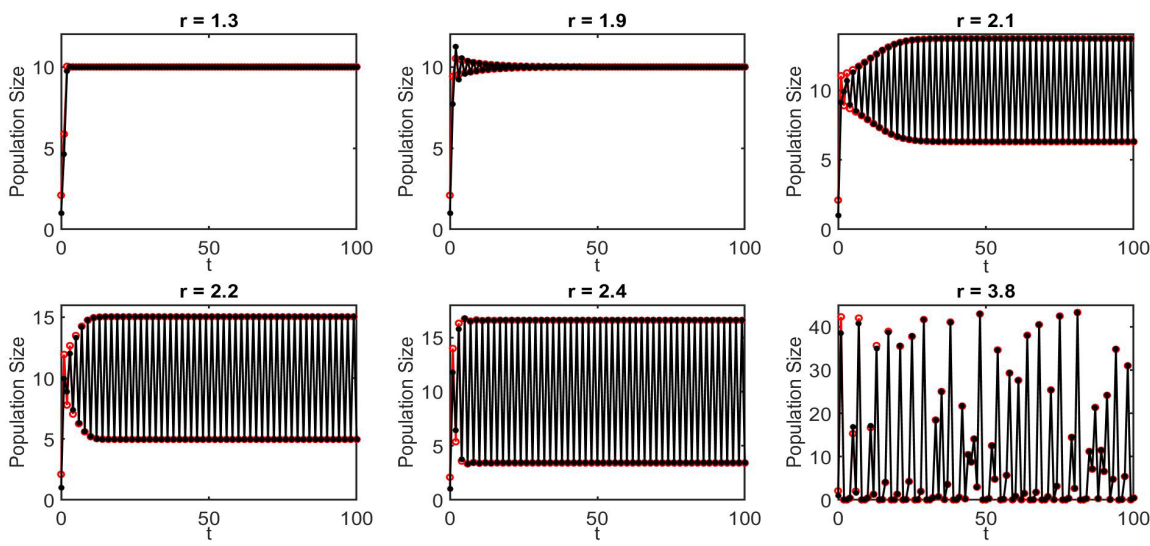

Figure 3. Evolution of host population $x_{1}$ and its coupled $x_{2}$ in time with two different initial conditions for drive-response system (3.1)-(3.2) when $s=0.5$ and $k=10$, drive system (red color) and response system (black color).

In Figure 4 and for the case $s=0.95$, we have almost the same dynamics as we had for $s=0.5$. As we can see, for larger values of threshold $s$, we do not get completely synchronized cycles.

Indeed, displayed dynamics in Figure 3 and Figure 4 are not special to the Ricker model, but are common features of discrete time population models.

For Figure 5, we explain some general properties which have been shared between the drive-response system (3.1)-(3.2) in common. As we can see, with increasing $r$ the value of carrying capacity $k$ is increasing. However, for $r \approx 2$ the branch corresponding to stable steady states bifurcates through period-doubling bifurcation into a 2-cycle. As we increase $r$ further, the interval over which we have a new period reduces, and as we know this smaller and smaller windows are called Feigenbaum cascade which after them the dynamics become aperiodic. As we expected, the bifurcation diagram of drive-response system (3.1)-(3.2) for greater values of $s$, shows the same types of dynamics for drive and response system, but not completely synchronized.

The Poincare section and power spectrum of drive-response system (3.1)-(3.2) have been displayed in Figure 6 for the case $s=0.95, r=3$ and $k=10$. Basically, the Poincare section can be constructed by sampling the phase portrait which helps to simplify the complicated dynamical systems. It is known that periodic behavior corresponds to a fixed point in Poincare section and any chaotic dynamics can be detected by set of distinct points in Poincare map. Moreover, the wideband chaotic signals and periodic signals can be easily distinguished from each other using the frequency spectra. Therefore, as we understood from bifurcation diagram, drive-response system (3.1)-(3.2) experiences the chaotic dynamics for $r=3$ and as we expected for large values of $s$ we can not establish a complete synchronization.

\section{Conclusion}

Synchronization in population dynamics can lead to arising complex dynamics and understanding the synchronization of oscillations is crucially important in 

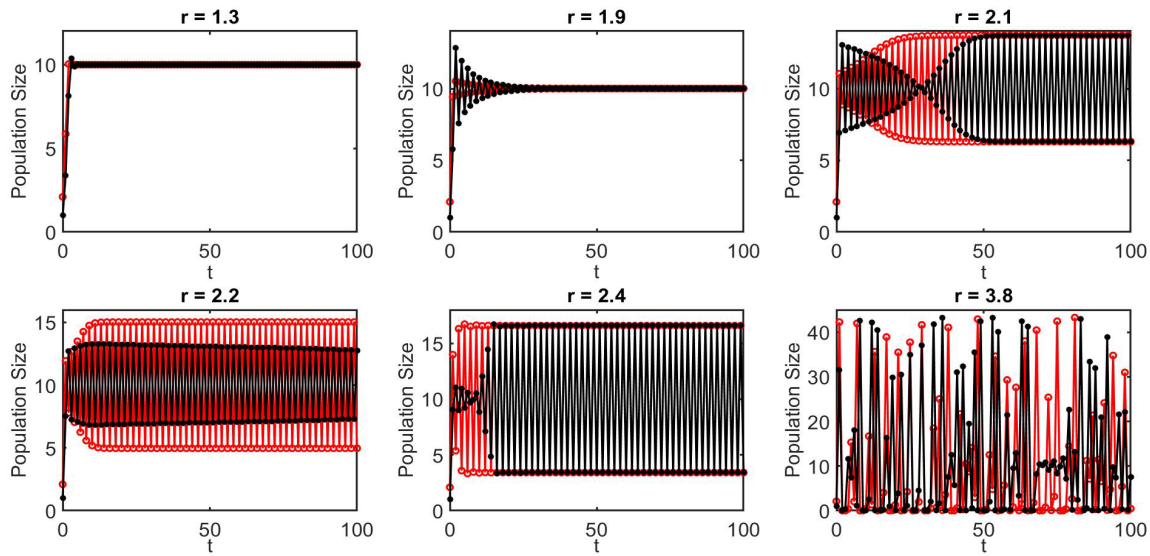

Figure 4. Evolution of host population $x_{1}$ and its coupled $x_{2}$ in time with two different initial conditions for drive-response system (3.1)-(3.2) when $s=0.95$ and $k=10$, drive system (red color) and response system (black color).
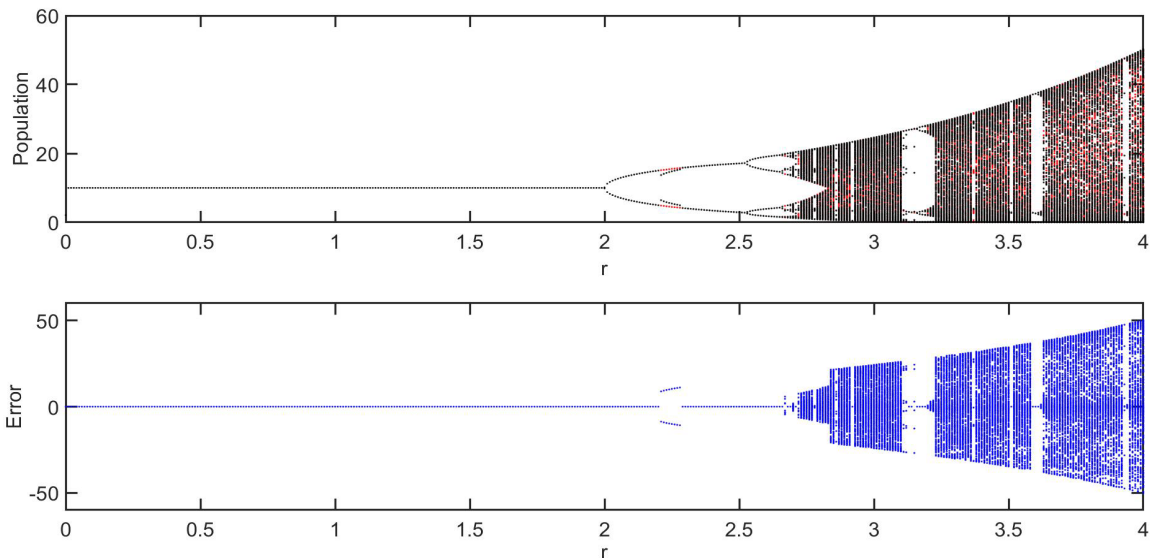

Figure 5. Bifurcation diagram of Ricker model and its coupled with the error between their attractors for $s=0.95$ and $k=10$, drive system (red color) and response system (black color).
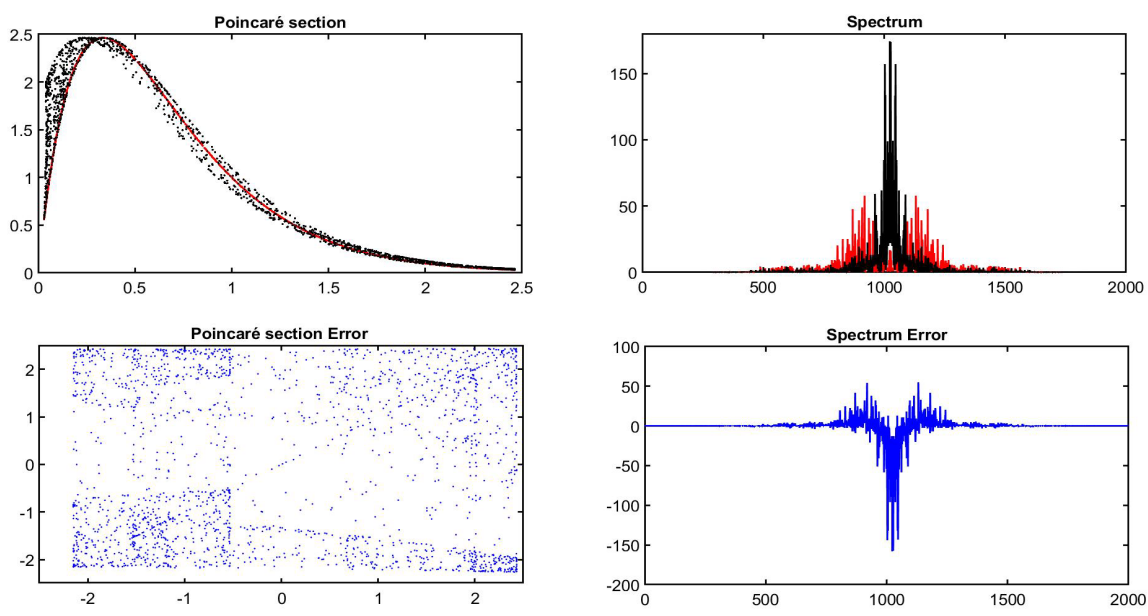

Figure 6. Poincare section and Spectrum for Ricker model and its coupled with corresponding error for $s=0.95, r=3$ and $k=10$, drive system (red color) and response system (black color). 
this area. In this study, we developed a new drive-response system by defining a convex continuous link function which maps the orbits of the drive system keeping the same qualitative properties such as stability and periodicity into the orbits of its coupled system. As has been shown by L. M. Pecora and T. L. Carroll, in 1990, two Lorenz systems with the property of sensitive dependence on the initial conditions could be synchronized starting from different initial states. We extended this result into discrete-time dynamical systems and we have shown that by using the concept of convex function, we can force the orbits of a discrete-time drive-response system starting from different initial conditions to get synchronized and we observed that this coupling method can be successful for drive-response system (3.1)-(.3.2) to get a complete synchronization when the synchronization threshold has smaller values, closer to zero. We also changed the values of synchronization threshold $s$ in its range between $(0,1)$ and we observed that the response system (3.2) for larger values of synchronization threshold $s$ is not completely synchronized with its original drive system (3.1) and when we increased the values of synchronization threshold $s$, we also noticed that the qualitative behaviors of both systems remain the same, even though, we do not get a complete synchronization between the solutions of drive and response system (3.1)-(3.2). In chaotic regime, for larger values of synchronization threshold $s$, closer to one, we could not get a complete synchronization. But, for smaller synchronization threshold $s$, closer to zero, we have shown that two systems are in complete synchronization even though the dynamics is chaotic.

\section{Acknowledgements}

This work was supported by the Institute of Computational Comparative Medicine (ICCM) and department of Mathematics of Kansas State University. With a special thanks to Dr. Majid Jaberi-Douraki for his full support.

\section{Conflicts of Interest}

The authors declare no conflicts of interest regarding the publication of this paper.

\section{References}

[1] Bellows, T.S. and Hassell, M.P. (1988) The Dynamics of Age-Structured Host-Parasitoid Interactions. The Journal of Animal Ecology, 57, 259-268. https://doi.org/10.2307/4777

[2] Hawkins, B.A. (2005) Pattern and Process in Host-Parasitoid Interactions. Cambridge University Press, Cambridge.

[3] Hawkins, B.A., Sheehan, W., et al. (1994) Parasitoid Community Ecology. Oxford University Press, Oxford.

[4] Earn, D.J.D., Rohani, P., Bolker, B.M. and Grenfell, B.T. (2000) A Simple Model for Complex Dynamical Transitions in Epidemics. Science, 287, 667-670. https://doi.org/10.1126/science.287.5453.667 
[5] Earn, D.J.D., Rohani, P., Bolker, B.G., Stefan, A.H. and Kisdi, É. (2004) On the Mechanistic Underpinning of Discrete-Time Population Models with Complex Dynamics. Journal of Theoretical Biology, 228, 261-269.

https://doi.org/10.1016/j.jtbi.2004.01.003

[6] Frisman, E.Y., Neverova, G.P. and Revutskaya, O.L. (2011) Complex Dynamics of the Population with a Simple Age Structure. Ecological Modelling, 222, 1943-1950.

https://doi.org/10.1016/j.ecolmodel.2011.03.043

[7] Zeng, Z., Nowierski, R.M., Taper, M.L., Dennis, B. and Kemp, W.P. (1998) Complex Population Dynamics in the Real World: Modeling the Influence of Time-Varying Parameters and Time Lags. Ecology, 79, 2193-2209. https://doi.org/10.1890/0012-9658(1998)079[2193:CPDITR]2.0.CO;2

[8] May, R.M. (1987) Chaos and the Dynamics of Biological Populations. Nuclear Physics B-Proceedings Supplements, 2, 225-245. https://doi.org/10.1016/0920-5632(87)90020-X

[9] Belykh, I., Hasler, M., Lauret, M. and Nijmeijer, H. (2005) Synchronization and Graph Topology. International Journal of Bifurcation and Chaos, 15, 3423-3433. https://doi.org/10.1142/S0218127405014143

[10] Cosp, J., Madrenas, J., Alarcón, E., Vidal, E. and Villar, G. (2004) Synchronization of Nonlinear Electronic Oscillators for Neural Computation. IEEE Transactions on Neural Networks, 15, 1315-1327. https://doi.org/10.1109/TNN.2004.832808

[11] Porfiri, M. and Pigliacampo, R. (2008) Master-Slave Global Stochastic Synchronization of Chaotic Oscillators. SIAM Journal on Applied Dynamical Systems, 7, 825-842. https://doi.org/10.1137/070688973

[12] Fujisaka, H. and Yamada, T. (1983) Stability Theory of Synchronized Motion in Coupled-Oscillator Systems. Progress of Theoretical Physics, 69, 32-47. https://doi.org/10.1143/PTP.69.32

[13] Pecora, L.M., Carroll, T.L., Johnson, G.A., Mar, D.J. and Heagy, J.F. (1997) Fundamentals of Synchronization in Chaotic Systems, Concepts, and Applications. Chaos. An Interdisciplinary Journal of Nonlinear Science, 7, 520-543. https://doi.org/10.1063/1.166278

[14] Pecora, L.M. and Carroll, T.L. (1990) Synchronization in Chaotic Systems. Physical Review Letters, 64, 821. https://doi.org/10.1103/PhysRevLett.64.821

[15] Boccaletti, S., Kurths, J., Osipov, G., Valladares, D.L. and Zhou, C.S. (2002) The Synchronization of Chaotic Systems. Physics Reports, 366, 1-101. https://doi.org/10.1016/S0370-1573(02)00137-0

[16] Li, G.-H. (2007) Modified Projective Synchronization of Chaotic System. Chaos, Solitons \& Fractals, 32, 1786-1790. https://doi.org/10.1016/j.chaos.2005.12.009

[17] Yassen, M.T. (2005) Chaos Synchronization between Two Different Chaotic Systems Using Active Control. Chaos, Solitons \& Fractals, 23, 131-140. https://doi.org/10.1016/j.chaos.2004.03.038

[18] Camazine, S., Deneubourg, J.-L., Franks, N.R., Sneyd, J., Bonabeau, E. and Theraula, G. (2003) Self-Organization in Biological Systems. Princeton University Press, Princeton, NJ.

[19] Tarai, A., Poria, S. and Chatterjee, P. (2009) Synchronization of Generalised Linearly Bidirectionally Coupled Unified Chaotic System. Chaos, Solitons \& Fractals, 40, 885-892. https://doi.org/10.1016/j.chaos.2007.08.039

[20] Kocarev, L. and Parlitz, U. (1996) Generalized Synchronization, Predictability, and Equivalence of Unidirectionally Coupled Dynamical Systems. Physical Review Let- 
ters, 76, 1816. https://doi.org/10.1103/PhysRevLett.76.1816

[21] Yang, T. and Chua, L.O. (1999) Generalized Synchronization of Chaos via Linear Transformations. International Journal of Bifurcation and Chaos, 9, 215-219. https://doi.org/10.1142/S0218127499000092

[22] Rosenblum, M.G., Pikovsky, A.S. and Kurths, J. (1996) Phase Synchronization of Chaotic Oscillators. Physical Review Letters, 76, 1804. https://doi.org/10.1103/PhysRevLett.76.1804

[23] Rosenblum, M.G., Pikovsky, A.S. and Kurths, J. (1997) From Phase to Lag Synchronization in Coupled Chaotic Oscillators. Physical Review Letters, 78, 4193. https://doi.org/10.1103/PhysRevLett.78.4193

[24] Khan, M.A. and Poria, S. (2012) Projective Synchronization of Bidirectionally Coupled Chaotic Systems via Linear Transformations. International Journal of Applied, 1, 541-548. https://doi.org/10.14419/ijamr.v1i4.332

[25] Mainieri, R. and Rehacek, J. (1999) Projective Synchronization in Three-Dimensional Chaotic Systems. Physical Review Letters, 82, 3042.

https://doi.org/10.1103/PhysRevLett.82.3042

[26] Kolmogorov, A.N. and Fomin, S.V. (1957) Elements of the Theory of Functions and Functional Analysis.

[27] Post, E. and Forchhammer, M.C. (2002) Synchronization of Animal Population Dynamics by Large-Scale Climate. Nature, 420, 168. https://doi.org/10.1038/nature01064

[28] Noble, A.E., Machta, J. and Hastings, A. (2015) Emergent Long-Range Synchronization of Oscillating Ecological Populations without External Forcing Described by Ising Universality. Nature Communications, 6, 6664. https://doi.org/10.1038/ncomms7664

[29] Manica, V. and Silva, J.A.L. (2014) Population Distribution and Synchronized Dynamics in a Metapopulation Model in Two Geographic Scales. Mathematical Biosciences, 250, 1-9. https://doi.org/10.1016/j.mbs.2014.02.002

[30] Gouhier, T.C., Guichard, F. and Menge, B.A. (2010) Ecological Processes Can Synchronize Marine Population Dynamics over Continental Scales. Proceedings of the National Academy of Sciences, 107, 8281-8286. https://doi.org/10.1073/pnas.0914588107

[31] Ricker, W.E. (1954) Stock and Recruitment. Journal of the Fisheries Board of Canada, 11, 559-623. https://doi.org/10.1139/f54-039

[32] Azizi, T., et al. (2015) Dynamics of a Discrete-Time Plant-Herbivore Model. Caspian Journal of Mathematical Sciences, 4, 241-256.

[33] Smith, H.L. and Thieme, H.R. (2011) Dynamical Systems and Population Persistence. American Mathematical Society, 118.

[34] Mormann, F., Lehnertz, K., David, P. and Elger, C.E. (2000) Mean Phase Coherence as a Measure for Phase Synchronization and Its Application to the EEG of Epilepsy Patients. Physica D: Nonlinear Phenomena, 144, 358-369. https://doi.org/10.1016/S0167-2789(00)00087-7

[35] Vinck, M., Oostenveld, R., Van Wingerden, M., Battaglia, F. and Pennartz, C.M.A. (2011) An Improved Index of Phase-Synchronization for Electrophysiological Data in the Presence of Volume-Conduction, Noise and Sample-Size Bias. NeuroImage, 55, 1548-1565. https://doi.org/10.1016/j.neuroimage.2011.01.055

[36] Daume, J., Gruber, T., Engel, A.K. and Friese, U. (2017) Phase-Amplitude Coupling and Long-Range Phase Synchronization Reveal Frontotemporal Interactions during 
Visual Working Memory. Journal of Neuroscience, 37, 313-322.

https://doi.org/10.1523/JNEUROSCI.2130-16.2016

[37] Lowet, E., Roberts, M.J., Bonizzi, P., Karel, J. and De Weerd, P. (2016) Quantifying Neural Oscillatory Synchronization: A Comparison between Spectral Coherence and Phase-Locking Value Approaches. PLoS ONE, 11, e0146443.

https://doi.org/10.1371/journal.pone.0146443

[38] Gambuzza, L.V., Gomez-Gardenes, J. and Frasca, M. (2016) Amplitude Dynamics Favors Synchronization in Complex Networks. Scientific Reports, 6, 24915. https://doi.org/10.1038/srep24915

[39] Liu, H. and Zhang, P. (2018) Phase Synchronization Dynamics of Neural Network during Seizures. Computational and Mathematical Methods in Medicine, 2018, 1-9. https://doi.org/10.1155/2018/7325938 